\title{
Typologie des systèmes d'élevage camelins du sud du Maroc
}

\author{
Asma Kamili ${ }^{1 *}$ Bernard Faye ${ }^{2,3 *}$ \\ Nour Saïd Tligui ${ }^{4}$ Mohammed Bengoumi ${ }^{5}$
}

\section{Mots-clés}

Camelus dromedarius, typologie, élevage, conduite d'élevage, Maroc

\author{
Submitted: 27 February 2019 \\ Accepted: 3 December 2019 \\ Published: 11 May 2020
}

DOI: $10.19182 /$ remvt.31862

\begin{abstract}
Résumé
Bien que disposant d'un appui des autorités nationales dans le cadre du Plan Maroc Vert, l'élevage camelin au Maroc est encore assez mal connu. La présente étude a eu pour objectif de disposer d'une description des types d'élevages camelins dans une portion vaste du sud du pays, caractérisée par sa grande concentration des élevages camelins. Pour ce faire, dans le cadre d'une enquête sanitaire, 168 éleveurs chameliers appartenant à neuf provinces du sud du Maroc ont été interviewés sur leur système de production. Les 168 troupeaux camelins ont représenté 11426 dromadaires soit environ $10 \%$ de la population cameline de cette zone. Au final, un tableau comprenant 168 élevages camelins décrits par 20 variables actives (taille et structure du troupeau, organisation de la gestion et pouvoir de décision, ampleur de la mobilité, commercialisation, pratiques de complémentation alimentaire et de prévention sanitaire) et deux variables illustratives (statut de l'enquêté et région d'origine) a été soumis à une analyse multivariée (analyse des correspondances multiples et classification automatique). L'analyse a permis d'identifier quatre types d'éleveurs : a) ceux possédant de grands troupeaux laitiers traditionnels, b) les citadins possédant des petits troupeaux, c) les éleveurs pluriactifs opportunistes, et d) les éleveurs parfois pluriactifs, faiblement intégrés au marché et sous-traitant leur activité d'élevage. Cette typologie paraît être très liée à la région d'origine des éleveurs indiquant une certaine spécificité régionale.
\end{abstract}

- Comment citer cet article: Kamili A., Faye B., Tligui N.S., Bengoumi M., 2020. Typology of camel farming systems in the south of Morocco. Rev. Elev. Med. Vet. Pays Trop., 73 (2): 71-80, doi: 10.19182/ remvt. 31862

\section{INTRODUCTION}

Bien que ne représentant que $3 \%$ de la biomasse herbivore domestique sur l'ensemble du territoire marocain, le dromadaire joue un rôle prépondérant dans les provinces sahariennes. La production de lait et de viande estimée respectivement à 6400 et 4600 tonnes (FAOstat consulté en 2019) représente plus de $95 \%$ du lait produit et consommé dans les régions de Dakhla et de Laâyoune, et $60 \%$ de la viande rouge. Ces proportions sont parmi les plus élevées dans les pays d'élevage camelin, en dépit d'une importance numérique modeste (170 000 têtes). Les dromadaires participent donc de façon

\footnotetext{
1. Office national de sécurité sanitaire des produits alimentaires, Rabat, Maroc. 2. CIRAD, UMR SELMET, F-34398 Montpellier, France.

3. SELMET, Univ Montpellier, CIRAD, INRAE, Institut Agro, Montpellier, France.

4. Institut agronomique et vétérinaire Hassan II, Rabat, Maroc.

5. Office régional, FAO, Tunisie.

*Auteur pour la correspondance

Email : asma_kamili@yahoo.fr
}

significative aussi bien aux revenus des éleveurs qu'à la sécurité alimentaire des populations sahraouies (El Abrak, 2000).

Vu son importance, ce secteur a bénéficié depuis les années 1990 d’un appui et d'un encadrement de l'état opéré par les services des Directions régionales de l'agriculture (DRA), les services vétérinaires de l'Office national de sécurité sanitaire des produits alimentaires (ONSSA) et les actions de l'Office national du conseil agricole (ONCA). La filière cameline est l'une des composantes majeures du Plan Maroc Vert démarré en 2010 dans les régions sahariennes. Cette filière bénéficie également d'autres programmes initiés par d'autres institutions dont l'Agence du Sud. En partenariat avec les organisations interprofessionnelles, un programme a été mis en place, visant notamment l'augmentation de la production de viande et de lait de chamelle.

Lélevage camelin est la plupart du temps de type pastoral extensif bien que de profondes mutations du pastoralisme camelin amènent à considérer que la catégorisation classique en nomade, transhumant ou sédentaire, ne soit ni opérationnelle, ni pertinente (Faye, 2018). La concentration accrue des élevages camelins laitiers dans la périphérie des villes sahariennes et des oasis en est l'expression la plus patente. Cette tendance à la périurbanisation s'appuie sur une sédentarisation souvent partielle du troupeau, de façon permanente ou transitoire 
selon les opportunités commerciales ou les contraintes climatiques (Faye et al., 2017). Une première typologie des élevages camelins de cette région avait été réalisée il y a une vingtaine d'années (Michel et al., 1997) montrant une faible diversité des pratiques à l'exception des pratiques de déplacement. Vingt ans plus tard et compte tenu des appuis au développement de cette filière mentionnés plus haut, se pose la question de l'évolution des systèmes de production camelins. L'objectif de cette étude était d'élaborer une typologie des systèmes camelins dans les régions du sud du Maroc afin d'en percevoir les évolutions, notamment sous l'effet des politiques de développement local.

\section{MATERIEL ET METHODES}

\section{Echantillonnage}

Afin de réaliser l'étude typologique, un échantillonnage stratifié a été établi dans le cadre d'un suivi sanitaire. Pour ce faire, les élevages ont été choisis dans neuf provinces connues pour l'importance de leur cheptel camelin, de telle manière que le nombre d'élevages sélectionnés était approximativement proportionnel au nombre d'éleveurs camelins répertoriés dans chaque province. Au total, 168 troupeaux ont été choisis dans les provinces de Boujdour (20), Dakhla (52), Essaouira (10), Guelmim (12), Laâyoune (19), Ouarzazate (14), Smara (12), Tan-Tan (19) et Tata (10). Ces troupeaux représentaient 11426 têtes de dromadaires, correspondant à un taux d'échantillonnage d'environ $10 \%$ de l'effectif total estimé du cheptel camelin dans ces régions du Maroc. La prise en compte du facteur région a permis d'intégrer une diversité de contextes reflétant a priori une diversité des systèmes d'élevage.

\section{Fiches d'enquête}

L'enquête a été réalisée sous la supervision et avec l'appui des services vétérinaires locaux qui ont procédé, avant de se déplacer, au repérage des troupeaux de dromadaires soit par contact du propriétaire par téléphone soit par la recherche des points d'eau et des lieux de rassemblement pastoral. Pour les besoins de cette enquête, deux fiches ont été élaborées :

une fiche " enquêté » pour chaque élevage camelin permettant de recueillir des données relatives au statut de l'enquêté, propriétaire ou non, permettant de connaître notamment son implication directe ou indirecte dans la gestion du troupeau ainsi que le niveau de prise de décision en matière de conduite d'élevage, auxquels ont été ajoutées les informations sur son appartenance tribale ;

une fiche «troupeau » visant à collecter les données relatives à la taille du troupeau, sa composition, son mode de renouvellement, la stratégie de ses déplacements, la gestion de l'alimentation, l'historique sanitaire, et les mesures préventives et médicales mises en œuvre pour la prévention ou le traitement des maladies.

\section{Préparation des données}

Les fiches renseignées ont été saisies sous Excel et les données ont été ensuite soumises à une recodification. Les variables qualitatives ont été éventuellement regroupées afin d'éviter les modalités rares, voire supprimées en l'absence de variabilité. La variable « effectif du troupeau », seule variable quantitative, a été transformée en une variable qualitative à trois modalités sur la base de la distribution des effectifs (tableau I). Les données concernant les écotypes présents dans

\section{Tableau I}

Variables et modalités retenues dans les analyses multivariées dans les élevages camelins du sud du Maroc, et distribution des modalités

\begin{tabular}{|c|c|c|c|c|c|}
\hline Variable & Acronyme & Modalité & Description & Effectif & $\%$ des modalités \\
\hline \multirow[t]{3}{*}{ Effectif du troupeau } & \multirow[t]{3}{*}{ Eff } & 1 & Petit troupeau & 35 & 20,83 \\
\hline & & 2 & Troupeau moyen & 61 & 36,31 \\
\hline & & 3 & Grand troupeau & 72 & 42,85 \\
\hline \multirow{3}{*}{ Personne interrogée } & \multirow[t]{3}{*}{ Pers } & 1 & Propriétaire et affiliés & 81 & 48,21 \\
\hline & & 2 & Contremaître & 31 & 18,45 \\
\hline & & 3 & Berger & 56 & 33,333 \\
\hline \multirow[t]{2}{*}{ Présence d'associés } & \multirow[t]{2}{*}{ Assoc } & 1 & Non & 141 & 83,93 \\
\hline & & 2 & Un ou plusieurs & 27 & 16,07 \\
\hline \multirow[t]{2}{*}{ Métier } & \multirow[t]{2}{*}{ Mét } & 1 & Eleveur uniquement & 76 & 45,24 \\
\hline & & 2 & Pluriactif & 92 & 54,76 \\
\hline \multirow[t]{2}{*}{ Habitation en ville } & \multirow[t]{2}{*}{ Vill } & 1 & Oui & 54 & 32,14 \\
\hline & & 2 & Non & 114 & 67,86 \\
\hline \multirow[t]{2}{*}{ Nb. de troupeaux } & \multirow[t]{2}{*}{ Nbtrp } & 1 & Un & 130 & 77,38 \\
\hline & & 2 & Plusieurs & 38 & 22,62 \\
\hline \multirow{4}{*}{$\begin{array}{l}\text { Nb. de visites du propriétaire } \\
\text { en saison sèche }\end{array}$} & \multirow[t]{4}{*}{ Vis } & 1 & Tous les jours & 95 & 56,55 \\
\hline & & 2 & Une à plusieurs fois par semaine & 34 & 20,24 \\
\hline & & 3 & Plusieurs fois par mois & 30 & 17,86 \\
\hline & & 4 & Plusieurs fois par saison & 9 & 5,36 \\
\hline \multirow[t]{3}{*}{$\mathrm{Nb}$. de bergers } & \multirow[t]{3}{*}{ Berg } & 1 & Aucun & 34 & 20,24 \\
\hline & & 2 & Un & 88 & 52,38 \\
\hline & & 3 & Plusieurs & 46 & 27,38 \\
\hline \multirow[t]{3}{*}{ Statut du berger } & \multirow[t]{3}{*}{ Stberg } & 1 & Famille & 64 & 38,09 \\
\hline & & 2 & Salarié & 87 & 51,78 \\
\hline & & 3 & Contrat & 17 & 10,12 \\
\hline
\end{tabular}




\section{Tableau I (suite)}

Variables et modalités retenues dans les analyses multivariées dans les élevages camelins du sud du Maroc, et distribution des modalités

\begin{tabular}{|c|c|c|c|c|c|}
\hline Variable & Acronyme & Modalité & Description & Effectif & $\%$ des modalités \\
\hline \multirow[t]{3}{*}{ Pouvoir de décision } & Dec & 1 & Aucun & 87 & 51,78 \\
\hline & & 2 & Alimentation & 10 & 5,95 \\
\hline & & 3 & Divers & 71 & 42,26 \\
\hline \multirow[t]{2}{*}{ Origine du berger } & Orig & 1 & Même origine & 95 & 56,55 \\
\hline & & 2 & Différent & 73 & 43,45 \\
\hline \multirow[t]{4}{*}{ Expérience du berger } & Exp & 1 & Faible & 36 & 21,43 \\
\hline & & 2 & Moyenne & 19 & 11,31 \\
\hline & & 3 & Elevée & 33 & 19,64 \\
\hline & & 4 & Non renseigné & 80 & 47,62 \\
\hline \multirow[t]{4}{*}{ Lieu achat/vente } & AV & 1 & Nomade & 11 & 6,55 \\
\hline & & 2 & Souk & 40 & 23,81 \\
\hline & & 3 & Souk et sur place & 40 & 23,81 \\
\hline & & 4 & Sur place & 77 & 45,83 \\
\hline \multirow[t]{2}{*}{ Effectifs achetés } & Ach & 1 & Non & 123 & 73,21 \\
\hline & & 2 & Oui & 45 & 26,78 \\
\hline \multirow[t]{3}{*}{ Déplacements } & Dep & 1 & Petit déplacement (< 100 km) & 22 & 13,09 \\
\hline & & 2 & Même endroit & 29 & 17,26 \\
\hline & & 3 & Grand déplacement (> 100 km ) & 117 & 69,63 \\
\hline \multirow[t]{2}{*}{ Structure troupeau } & Strp & 1 & Camelin & 143 & 85,12 \\
\hline & & 2 & Mixte & 25 & 14,88 \\
\hline \multirow[t]{3}{*}{ Introduction d'animaux } & Int & 1 & Non & 22 & 13,09 \\
\hline & & 2 & Oui & 139 & 82,74 \\
\hline & & 3 & Non renseigné & 7 & 4,17 \\
\hline \multirow[t]{4}{*}{ Vaccin variole } & Var & 1 & Jamais & 51 & 30,36 \\
\hline & & 2 & Occasionnellement & 39 & 23,21 \\
\hline & & 3 & Annuellement & 73 & 43,45 \\
\hline & & 4 & Non renseigné & 5 & 2,97 \\
\hline \multirow[t]{8}{*}{ Alimentation supplémentaire } & Alim & 1 & Betterave + céréales & 69 & 41,07 \\
\hline & & 2 & Céréales & 9 & 5,37 \\
\hline & & 3 & Dattes + céréales & 10 & 5,95 \\
\hline & & 4 & Farine + céréales & 10 & 5,95 \\
\hline & & 5 & Luzerne + céréales & 12 & 7,14 \\
\hline & & 6 & Mais + céréales & 11 & 6,55 \\
\hline & & 7 & Orge & 22 & 13,09 \\
\hline & & 8 & Rien & 25 & 14,88 \\
\hline \multirow[t]{6}{*}{ Bénéficiaires } & Ben & 1 & Animaux faibles & 11 & 6,55 \\
\hline & & 2 & Allaitantes & 10 & 5,95 \\
\hline & & 3 & Gestantes & 5 & 2,97 \\
\hline & & 4 & Mâles & 5 & 2,97 \\
\hline & & 5 & Non renseigné & 25 & 14,88 \\
\hline & & 6 & Tous & 112 & 66,66 \\
\hline \multirow[t]{2}{*}{ Médicaments } & Med & 1 & Oui & 148 & 88,09 \\
\hline & & 2 & Non & 20 & 11,90 \\
\hline \multirow[t]{9}{*}{ Province } & Prov & Boujdour & & 20 & 11,90 \\
\hline & & Dakhla & & 52 & 30,95 \\
\hline & & Essaouira & & 10 & 5,95 \\
\hline & & Guelmim & & 12 & 7,14 \\
\hline & & Laâyoune & & 19 & 11,31 \\
\hline & & Ouarzazate & & 14 & 8,33 \\
\hline & & Smara & & 12 & 7,14 \\
\hline & & Tan-Tan & & 19 & 11,31 \\
\hline & & Tata & & 10 & 5,95 \\
\hline
\end{tabular}


les troupeaux n'ont pas été introduites dans les variables destinées à l'analyse multivariée. Elles ont été mobilisées pour la description de l'échantillon dans son ensemble.

\section{Analyse statistique}

L'analyse typologique s'est appuyée sur les analyses multivariées classiques impliquant : a) le choix des variables actives et supplémentaires, b) une première analyse factorielle des correspondances multiples (AFCM) afin de tester les relations entre variables, c) une classification ascendante hiérarchique (CAH) permettant d'identifier les groupes d'élevages homogènes, et d) la construction d'un tableau de contingence croisant les classes issues de la CAH et chacune des variables qualitatives afin de déterminer les variables ayant une contribution significative aux classes (test du chi²). Les variables contribuant significativement aux classes ont permis de décrire les classes définissant les «types d'élevage ».

L'AFCM a été réalisée sur un tableau (i*j) comprenant 168 élevages camelins (i) et 20 variables actives (j), plus deux variables illustratives, à savoir la province d'origine et le statut de l'éleveur. La classification a porté sur les lignes du tableau des cinq premiers facteurs (soit $36 \%$ de l'inertie cumulée) en utilisant la méthode d'agrégation de Ward et la distance euclidienne (Everitt et al., 2011). Le logiciel utilisé a été XLstat (Addinsoft version 2019).

\section{RESULTATS}

\section{Profil des enquêtés}

Sur l'ensemble de l'échantillon, près de la moitié $(48,2 \%)$ des enquêtés étaient les propriétaires du troupeau camelin, ce troupeau pouvant être divisé en plusieurs sous-ensembles. Le propriétaire pouvait soit assurer lui-même toutes les activités de gardiennage, de déplacement et d'alimentation, soit disposer d'un ou de plusieurs bergers (salarié ou membre de sa famille) aux pouvoirs de décision variables. Par ailleurs, une part importante de ces propriétaires (68\%) étaient des pluriactifs, c'est-à-dire pratiquant une autre activité que l'élevage camelin (commerce, fonction publique notamment). De plus, $73 \%$ des propriétaires interrogés vivaient en ville, confiant leurs troupeaux à des bergers ou à un contremaître. Dans 18,4\% des cas, l'enquêté était un contremaître, donc a priori avec un pouvoir de décision plus large qu'un simple berger. Enfin, un tiers des enquêtés (33,3\%) étaient des bergers. L'élevage de dromadaires est un élément de base de la culture de la population marocaine, sahraouie en particulier. Or, cette population se caractérise par son organisation tribale. Au cours de notre étude, cette information a été collectée et 52 tribus ont pu être recensées selon les origines des propriétaires avec une prédominance des tribus de Aït Lahcen, Ouled Tidarine, Ezzargui, Rguibat, Ouled Bouaila, Laaroussine et Yegoth. Cette donnée n'a toutefois pas été intégrée dans l'analyse multivariée compte tenu du grand nombre de modalités.

\section{Description générale des troupeaux camelins}

La taille des troupeaux étudiés a varié d'une tête à Essaouira comme minimum à 256 têtes comme maximum à Dakhla. La taille moyenne des troupeaux a été de $68 \pm 54$ têtes, ce qui témoignait d'une assez grande variabilité. La médiane était de 50 têtes. Cette variabilité s'avère d'abord régionale. En effet, la taille moyenne des troupeaux a varié de 8 à Essaouira à 92 à Dakhla (figure 1). Sur l'ensemble de l'échantillon, la répartition des troupeaux en fonction de leur taille a permis de faire ressortir trois groupes relativement homogènes (figure 2) : a) les petits troupeaux, avec un effectif inférieur ou égal à 50 têtes, constituant $51 \%$ des troupeaux, b) les troupeaux de taille moyenne, de 51 à 130 têtes représentant $37 \%$ de l'échantillonnage, et c) les grands troupeaux, dont l'effectif était compris entre 131 et 256 têtes, représentant environ $12 \%$ des troupeaux. Cette distribution a permis de créer une variable qualitative « effectif du troupeau » (Eff) à trois modalités avec une surreprésentation des petits troupeaux.

Concernant les écotypes présents, dans les troupeaux, la notion de race étant peu applicable dans le cas des grands camélidés (Burger et al., 2019), la présente étude s'est appuyée sur la nomenclature proposée par le Groupe d'études et de recherches pour le développement de l'élevage camelin (Gerdec) dans les années 1990 (Ouassat et Achaabane, 1991). C'est ainsi que $46 \%$ des dromadaires de l'échantillon ont été rattachés au type Marmouri, $10 \%$ au type Guerzni, et le restant (44\%) n'a pu être affecté à un type déterminé. Le dromadaire Marmouri est un animal longiligne au poil ras de robe brun clair et avec une mamelle bien développée. A l'inverse, le dromadaire Guerzni est plutôt bréviligne, au poil plus abondant, mais sans couleur de robe très spécifique. C'est plutôt un animal de bât. Ces écotypes apparaissent cependant comme des subdivisions purement phénotypiques d'un seul génotype dit Sahraoui, largement répandu dans toute l'Afrique du Nord (Oulad Belkhir et al., 2013).

\section{Analyses multivariées}

L'AFCM a été réalisée sur la base des variables listées dans le tableau I. L'examen de l'histogramme des valeurs propres a indiqué

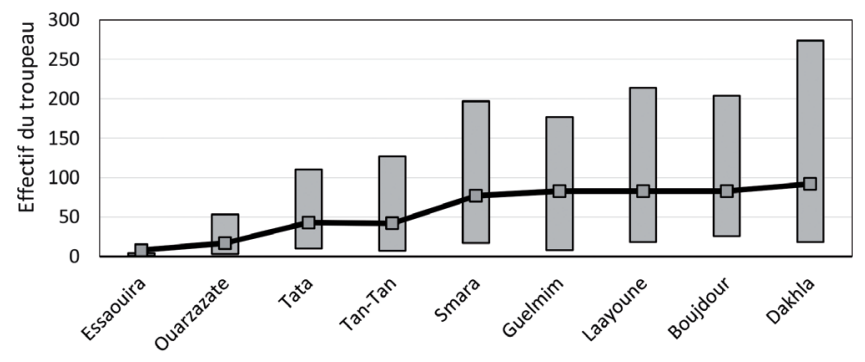

Figure 1 : moyenne (ligne) et minimum-maximum (histogramme) des effectifs des troupeaux camelins dans neuf provinces du Maroc, classés par ordre d'importance de la valeur moyenne.

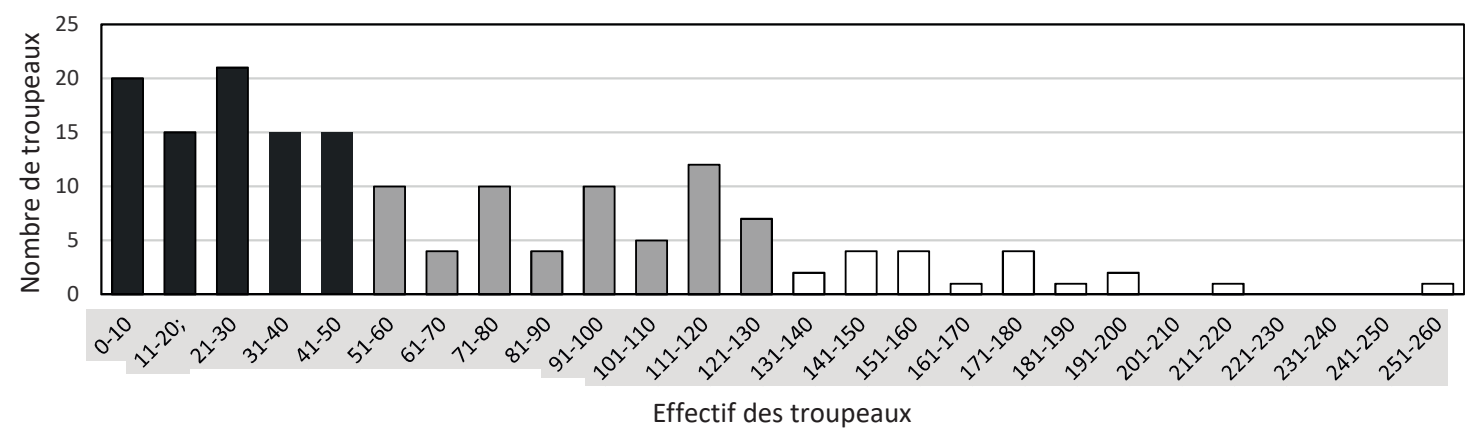

Figure 2 : répartition des troupeaux en fonction des effectifs camelins (Sud Maroc) par classe de dix têtes. 
un facteur F1 prépondérant exprimant $12,6 \%$ de l'inertie totale, soit huit fois la moyenne des valeurs propres. Le second facteur $(7,4 \%)$ et le troisième facteur $(6,2 \%)$ ont été retenus dans l'interprétation des relations entre variables, les gains d'inertie avec les facteurs suivants apparaissant mineurs.

Les modalités contribuant significativement au premier facteur ont permis d'identifier une opposition nette entre : a) les éleveurs citadins avec petits troupeaux (Eff1, Met1, Vill1) dont le troupeau unique est gardé par un membre de la famille (Nbtrp1, Stberg1) et qui se déplaçait sur des distances moyennes ou courtes (Dep1, Dep2) ; ces éleveurs distribuaient des céréales en complémentation (Alim3, Alim4, Alim6) à tous les animaux (Ben6), mais n'assuraient pas de protection sanitaire
(Var1, Med2) en dépit de visites régulières du propriétaire (Vis1) ; et b) les éleveurs pastoraux pluriactifs avec grands troupeaux (Eff3, Met2, Vill2) confiant la gestion de leurs troupeaux à des bergers salariés plus ou moins expérimentés ou à des contremaîtres (Stberg2, Exp1, Exp3, Dec3), avec des troupeaux se déplaçant sur de longues distances (Dep3); la complémentation était limitée aux animaux faibles à partir des aliments disponibles (Ben1, Alim4, Alim8) ; les animaux étaient vaccinés annuellement contre la variole, et le recours aux médicaments vétérinaires était la règle (Var3, Med2). Les premiers apparaissaient plutôt associés aux propriétaires des régions de Ouarzazate et d'Essaouira, les seconds étaient surtout des bergers et contremaîtres originaires de celle de Dakhla (tableau II).

\section{Tableau II}

Typologie d'élevages camelins du sud du Maroc - Valeurs-tests des contributions des modalités aux trois premiers facteurs de I'AFCM

\begin{tabular}{|c|c|c|c|}
\hline Modalité & F1 & F2 & F3 \\
\hline Eff1 & $7,670^{*}$ & $1,977^{*}$ & 0,543 \\
\hline Eff2 & 0,062 & 1,005 & $-5,215^{*}$ \\
\hline Eff3 & $-6,354^{*}$ & $-2,598^{*}$ & $4,622^{*}$ \\
\hline Assoc1 & 0,237 & $2,544^{*}$ & $-5,858^{*}$ \\
\hline Assoc2 & $-0,237$ & $-2,544^{*}$ & $5,858^{*}$ \\
\hline Mét1 & $5,890^{*}$ & $-4,848^{*}$ & $-2,256^{*}$ \\
\hline Mét2 & $-5,890^{*}$ & $4,848^{*}$ & $2,256^{*}$ \\
\hline Vill1 & $4,155^{*}$ & $-1,426$ & $-1,200$ \\
\hline Vill2 & $-4,155^{*}$ & 1,426 & 1,200 \\
\hline Nbtrp1 & $2,580^{*}$ & $2,347^{*}$ & $-2,377^{*}$ \\
\hline Nbtrp2 & $-2,580^{*}$ & $-2,347^{*}$ & $2,377^{*}$ \\
\hline Vis1 & $7,303^{*}$ & $-1,433$ & $-0,511$ \\
\hline Vis2 & $-2,135^{*}$ & $-0,637$ & 1,561 \\
\hline Vis3 & $-5,787^{*}$ & $2,122^{*}$ & $-1,369$ \\
\hline Vis4 & $-2,424^{*}$ & 0,680 & 0,667 \\
\hline Berg1 & $7,547^{*}$ & $-3,309 *$ & $-4,372^{*}$ \\
\hline Berg2 & $-5,070^{*}$ & $5,088^{*}$ & $-0,793$ \\
\hline Berg3 & $-1,122$ & $-2,717^{*}$ & $4,827^{*}$ \\
\hline Stberg1 & $8,093^{*}$ & $-3,551^{*}$ & $-4,962 *$ \\
\hline Stberg2 & $-7,338^{*}$ & $6,295^{*}$ & 1,226 \\
\hline Stberg3 & $-0,874$ & $-4,712^{*}$ & $5,958^{*}$ \\
\hline Dec1 & 1,257 & $-1,440$ & $-4,536 *$ \\
\hline Dec2 & $5,876^{*}$ & $9,479 *$ & $3,727^{*}$ \\
\hline Dec3 & $-4,087^{*}$ & $-3,083^{*}$ & $2,803^{*}$ \\
\hline Orig1 & $9,985^{*}$ & $-2,482^{*}$ & $-1,234$ \\
\hline Orig2 & $-9,985^{*}$ & $2,482^{*}$ & 1,234 \\
\hline Exp1 & $-4,937^{*}$ & 1,608 & $-2,370^{*}$ \\
\hline Exp2 & $-2,100 *$ & $-1,377$ & $2,302^{*}$ \\
\hline Exp3 & $-3,639 *$ & $-1,098$ & $3,007^{*}$ \\
\hline Exp4 & $8,283^{*}$ & 0,425 & $-1,904$ \\
\hline AV1 & $6,228^{*}$ & $9,301^{*}$ & $3,579 *$ \\
\hline AV2 & $3,898^{*}$ & $-4,237^{*}$ & $2,409 *$ \\
\hline AV3 & $2,584^{*}$ & $-4,801^{*}$ & $-2,953^{*}$ \\
\hline AV4 & $-8,632^{*}$ & $3,108^{*}$ & $-1,312$ \\
\hline Ach1 & $-2,258^{*}$ & $3,225^{*}$ & $-5,047^{*}$ \\
\hline Ach2 & $2,258^{*}$ & $-3,225^{*}$ & $5,047^{*}$ \\
\hline Dep1 & $2,478^{*}$ & $-2,399 *$ & $-2,217^{*}$ \\
\hline Dep2 & $8,591^{*}$ & $4,624^{*}$ & $2,042^{*}$ \\
\hline Dep3 & $-8,879^{*}$ & $-2,040^{*}$ & $-0,052$ \\
\hline
\end{tabular}

\begin{tabular}{|c|c|c|c|}
\hline Modalité & $\mathrm{F} 1$ & F2 & F3 \\
\hline Strp1 & $-0,276$ & 1,419 & $4,391^{*}$ \\
\hline Strp2 & 0,276 & $-1,419$ & $-4,391 *$ \\
\hline Int1 & $-3,534^{*}$ & 1,667 & $-1,929$ \\
\hline Int2 & $2,201^{*}$ & 0,170 & 1,835 \\
\hline Int3 & 1,803 & $-3,136^{*}$ & $-0,214$ \\
\hline Var1 & $7,629^{*}$ & $3,990^{*}$ & $-2,424^{*}$ \\
\hline Var2 & 1,336 & $-6,306^{*}$ & $4,359 *$ \\
\hline Var3 & $-8,489 *$ & 1,942 & $-1,284$ \\
\hline Var4 & 0,800 & $-0,792$ & $-0,525$ \\
\hline Alim1 & $-2,244^{*}$ & $-2,769^{*}$ & 1,002 \\
\hline Alim2 & 0,901 & $-3,762 *$ & $2,301^{*}$ \\
\hline Alim3 & $5,277^{*}$ & $8,446^{*}$ & $3,137^{*}$ \\
\hline Alim4 & $-3,409^{*}$ & 1,577 & $2,617^{*}$ \\
\hline Alim5 & $2,971^{*}$ & 0,022 & 1,922 \\
\hline Alim6 & $3,832^{*}$ & $-0,012$ & $-1,492$ \\
\hline Alim7 & $-0,673$ & $-1,456$ & 0,571 \\
\hline Alim8 & $-2,887^{*}$ & 0,917 & $-7,561^{*}$ \\
\hline Ben1 & $-3,491^{*}$ & 1,668 & $-1,050$ \\
\hline Ben2 & $-0,090$ & $-1,233$ & $-2,101^{*}$ \\
\hline Ben3 & $-1,170$ & $-0,472$ & 0,937 \\
\hline Ben4 & $-2,237^{*}$ & $2,036^{*}$ & $-0,643$ \\
\hline Ben5 & $-2,887^{*}$ & 0,917 & $-7,561^{*}$ \\
\hline Ben6 & $5,285^{*}$ & $-1,513$ & $7,207^{*}$ \\
\hline Med1 & $-2,907^{*}$ & $2,332^{*}$ & $-0,353$ \\
\hline Med2 & $2,907^{*}$ & $-2,332 *$ & 0,353 \\
\hline \multicolumn{4}{|c|}{ Variables supplémentaires } \\
\hline Pers.1 & $6,455^{*}$ & $-2,926^{*}$ & $-1,412$ \\
\hline Pers.2 & $-2,289 *$ & $2,038^{*}$ & 1,443 \\
\hline Pers.3 & $-4,959 *$ & 1,424 & 0,310 \\
\hline Boujdour & $-1,601$ & $-0,943$ & $-0,505$ \\
\hline Dakhla & $-9,245^{*}$ & $4,658^{*}$ & $-1,779$ \\
\hline Essaouira & $5,011^{*}$ & $-0,538$ & $-1,515$ \\
\hline Guelmim & 1,279 & $-4,325^{*}$ & $2,890^{*}$ \\
\hline Laâyoune & 0,328 & $-3,873^{*}$ & $3,718^{*}$ \\
\hline Ouarzazate & $6,313^{*}$ & $8,505^{*}$ & $3,481^{*}$ \\
\hline Smara & 0,315 & $-1,736$ & $-2,804 *$ \\
\hline Tan-Tan & $2,962^{*}$ & $-3,218^{*}$ & $-1,425$ \\
\hline Tata & 1,729 & $-1,117$ & $-1,548$ \\
\hline
\end{tabular}


Le second facteur faisait surtout ressortir les variables liées à la commercialisation et à certaines formes de complémentation alimentaire. C'est ainsi que les éleveurs pratiquant l'achat d'animaux directement chez les nomades (AV1) s'opposaient à ceux qui pratiquaient les achats dans les souks ou sur place (AV2 et AV3) ; les premiers n'étaient pas vendeurs (Ach1), contrairement aux seconds (Ach2) ; les premiers supplémentent avec des dattes et des céréales, les seconds distribuaient des betteraves et des céréales (Alim1, Alim2). Les premiers étaient plutôt originaires de la province de Ouarzazate, et les seconds se localisaient plutôt dans les provinces de Guelmim, Tan-Tan et Laâyoune.

Le troisième facteur était structuré autour de l'opposition entre a) les éleveurs chameliers spécialisés (Strp1) disposant d'un berger salarié (Stberg3) avec pouvoir de décision (Dec2, Dec3) pratiquant une complémentation alimentaire diversifiée à tous les animaux (Ben6), et b) les éleveurs de troupeaux pluriespèces (Strp2) sans berger extérieur à la famille (Berg1, Stberg1), ou ne disposant pas de pouvoir de décision (Dec1) et pratiquant une supplémentation alimentaire mal définie (Alim8, Ben5). Leur répartition géographique était plus diffuse, mais les premiers provenaient plutôt de Laâyoune et de Guelmim, et les seconds plutôt de Smara. Il y avait globalement une distribution régionale marquée avec notamment une nette différence entre les élevages de la région de Ouarzazate et ceux de Dakhla (figure 3).

L'étape suivante de l'analyse basée sur le regroupement des élevages selon leur ressemblance a permis d'identifier quatre groupes bien distincts (décrits ci-après), comme l'indique l'arbre de classification (figure 4), avec une variance interclasses de 53,2\%.

Le type 1, les «Eleveurs de grands troupeaux », le plus souvent vivant dans le désert, était le groupe comprenant le plus d'éleveurs ( $\mathrm{n}=67$ soit près de $40 \%$ ). Ces derniers pouvaient disposer d'associés et de bergers salariés expérimentés provenant de la région. Le propriétaire achetait des animaux pour accroître son cheptel en se les procurant plutôt au souk. Concernant les déplacements, le groupe était hétérogène pratiquant une mobilité sur de courtes ou de longues distances. Le chamelier vaccinait ses animaux occasionnellement et distribuait à tous les animaux un complément alimentaire à base de pulpe de betterave et de céréales. Ces chameliers, majoritairement des provinces de Tan-Tan, Boujdour et Guelmim, accessoirement de Laâyoune, étaient des éleveurs chameliers de grands troupeaux,

Observations (axes F1 et F2 : $19,96 \%$ )

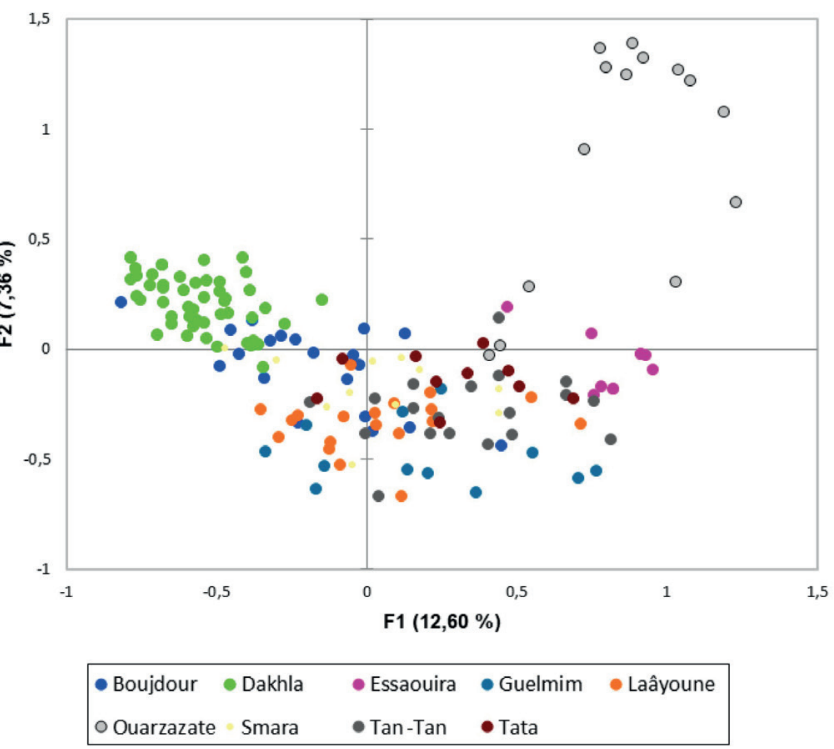

Figure 3 : répartition des élevages camelins (Sud Maroc) dans le plan factoriel $(1,2)$, identifiés selon leur localisation provinciale. pratiquant un élevage traditionnel et prenant soin des animaux, dont la vocation est laitière.

Le type 2, «Eleveurs familiaux avec petits troupeaux » $(\mathrm{n}=37$ soit $22 \%$ des éleveurs). Ces éleveurs ne faisaient pas toujours appel à des bergers, ou alors à des membres de la famille, souvent habitant en ville. Ils pratiquaient des déplacements sur de faibles ou moyennes distances et vendaient leurs animaux au souk. Les animaux n'étaient pas vaccinés et la supplémentation destinée à toutes les catégories d'animaux contenait des dattes et des céréales, parfois de la luzerne et du maïs. Ces chameliers en provenance surtout de Ouarzazate, de Tata et d'Essaouira étaient des éleveurs familiaux en majorité citadins n'ayant pas d'autre activité que l'élevage et possédaient un petit troupeau.

Le type 3, «Eleveurs pluriactifs », en majorité n'habitait cependant pas en ville. C'était le groupe le moins nombreux $(n=22$ soit $13 \%)$. Ces éleveurs recrutaient des bergers salariés ou un contremaître qui se déplaçaient sur de longues distances avec les dromadaires. Les animaux étaient vaccinés annuellement. La supplémentation, contenant surtout de la pulpe de betterave et des céréales, n'était distribuée qu'aux animaux les plus faibles et parfois aux chamelles allaitantes ou gestantes. Ces éleveurs étaient plutôt originaires de Boudjour, Tata, Laâyoune ou Smara. Ils étaient pluriactifs et relativement opportunistes sur la gestion de leur troupeau en termes de délégation de pouvoir de décision et de complémentation des animaux.

Le type 4, «Eleveurs chameliers parfois pluriactifs ayant un troupeau de taille moyenne » $(n=42$, soit $25 \%$ des éleveurs). Ces éleveurs vivaient dans le désert, généralement associé à d'autres. Le troupeau était géré par un seul berger originaire de l'extérieur de la province, mais ayant un large pouvoir décisionnel même si le propriétaire était souvent présent. Les troupeaux effectuaient de longs déplacements et étaient en général vaccinés annuellement contre la variole cameline. Il n'y avait pas eu d'achats récents d'animaux et ces éleveurs commerçaient en dehors des souks. La supplémentation était à base d'orge et de divers sous-produits destinés surtout aux dromadaires mâles, mais dans un certain nombre de cas il n'y avait pas de supplémentation. Ces chameliers étaient surtout originaires de Dakhla et dans une moindre mesure de Smara et Laâyoune. Ils étaient parfois pluriactifs, sous-traitant leur activité d'élevage, avec une intégration au marché relativement faible.

Les variables «nombre de troupeaux », « structure du troupeau », « introduction des animaux », et « médicaments » n'ont pas contribué

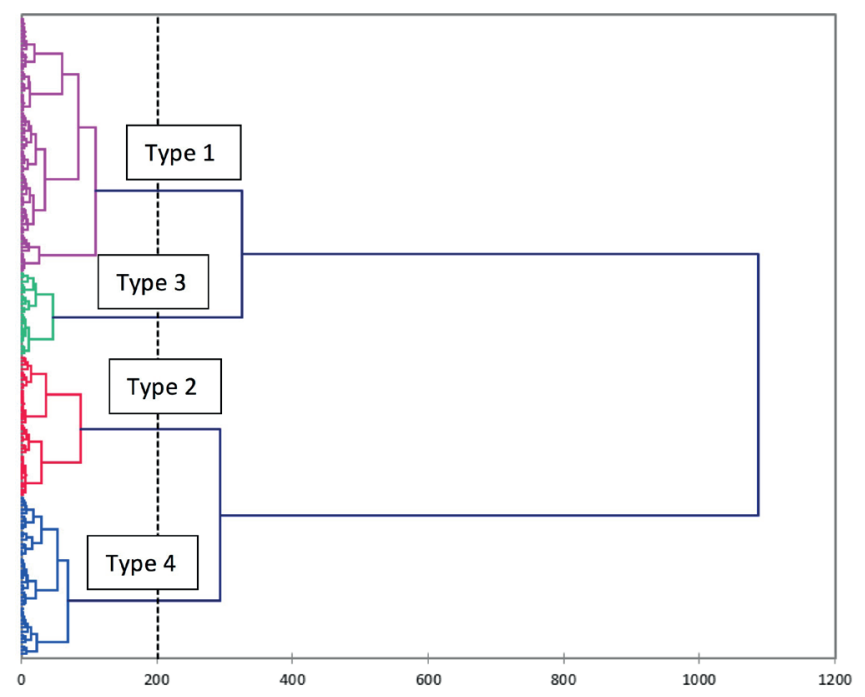

Figure 4 : arbre de classification des 168 élevages camelins enquêtés dans les provinces du sud du Maroc. 
significativement à la constitution des types d'élevage. Le lien entre les classes ainsi identifiées et les provinces d'origine peut être visualisé dans un tableau de contingence croisant ces deux variables illustrant la répartition géographique des types d'élevage (tableau III).

\section{DISCUSSION}

Il existe peu de données sur les systèmes d'élevage camelins présents en Afrique du Nord, notamment au Maroc. Des typologies d'élevage sont disponibles en Algérie (Adamou, 2008 ; Bedda et al., 2015), en Tunisie (Salmi et al., 2016) et pour les parties sahariennes du Maroc (Faye et al., 2014). Les données relatives aux systèmes de production camelins dans les provinces du sud du Maroc sont rares et les études n'ont été réalisées que sur quelques troupeaux et dans la seule région de Laâyoune (Michel et al., 1997).

Le présent travail est le résultat d'une enquête réalisée dans les neuf provinces situées au sud du Maroc, où se concentre $75 \%$ du cheptel camelin national des dromadaires. Les rubriques abordées dans le questionnaire constituent une partie des critères habituellement utilisés pour définir les systèmes d'élevage pastoraux dans cette région. En effet, selon Faye et al. (2014), la taille des troupeaux, le degré de mobilité, le degré d'intensification alimentaire, le mode de gardiennage et le degré d'intégration marchande renseignent le gradient d'intensification que l'on peut observer dans ces systèmes. Toutefois, notre questionnaire n'a pas abordé les pratiques liées à la production (traite, reproduction), à la gestion des effectifs (réforme, entrées-sorties) ni aux performances zootechniques, ce qui constitue de fait la limite de l'enquête. La collecte de telles données aurait nécessité des suivis (enquêtes longitudinales) plus difficiles et plus coûteux à mettre en place. Il aurait été possible cependant de disposer de données quantitatives déclaratives (volumes de vente, quantités de lait produit), mais leur fiabilité est souvent discutable (Faye et al., 1994).

\section{Taille des troupeaux}

Dans l'étude de Michel et al. (1997) dans la province de Laâyoune, les observations portent sur 170 éleveurs représentant 12000 dromadaires (soit $44 \%$ des dromadaires recensés). Dans cette étude, la taille des troupeaux camelins est comprise entre 2 et 900 dromadaires
(79 \pm 106 animaux par troupeau en moyenne, avec une médiane également de 50 individus), soit des chiffres assez comparables à ceux observés dans notre échantillonnage géographique élargi. Toutefois, la distribution des effectifs entre petits, moyens et grands troupeaux diffère sensiblement de celle observée dans notre étude. En effet, plus de $50 \%$ des troupeaux de la région de Laâyoune comptent entre 20 et 95 dromadaires.

\section{Propriétaires citadins et bergers sur les parcours}

Dans notre étude, les types se distinguaient nettement sur l'urbanité ou non du propriétaire. Ainsi, $55 \%$ des éleveurs du type 2 étaient des citadins. Dans l'étude de Michel et al. (1997) ciblée sur la seule région de Laâyoune, cette proportion est de $25 \%$, et la fréquence de leurs visites au troupeau est extrêmement variable. Il s'agit d'un phénomène que l'on retrouve ailleurs dans les pays du golfe (Abdallah et Faye, 2013) ; il est souvent associé à la multiactivité des propriétaires (Faye, 2016) ainsi qu'au mode de vie des éleveurs qui a évolué avec une tendance à la sédentarisation en substitution du déplacement avec la famille sur les parcours où les conditions de confort sont réduites.

Cette observation a été faite également chez les éleveurs de dromadaires en Algérie. En effet, Titaouine et al. (2015) rapportent que le lieu de résidence des éleveurs confirme la tendance actuelle vers la sédentarisation en milieu urbain ou au village puisque plus de $61 \%$ d'entre eux partagent leur vie entre le village et leur tribu tandis que $16,6 \%$ sont résidents dans leur village. La tendance à la « périurbanisation » de l'élevage camelin est attestée, notamment autour des agglomérations oasiennes, dans l'ensemble saharien et sahélo-saharien (Faye et al., 2017).

Ce changement dans le mode de vie des éleveurs chameliers explique que, dans notre étude, les troupeaux étaient dans $61 \%$ des cas sous la responsabilité d'un berger ou d'un contremaitre, conformément aux observations faites vingt ans plus tôt (Michel et al., 1997). Notons que dans le Sud Est algérien, le nombre de bergers ne représente que $5 \%$ du nombre total d'éleveurs enquêtés par Titaouine et al. (2015) du fait des difficultés à trouver des bergers de confiance, obligeant le propriétaire à assumer les tâches de gardiennage. Cette contrainte pourrait, par ailleurs, expliquer la pratique courante en Algérie de la divagation (troupeau sans gardiennage) qui porte le nom de hmil

\section{Tableau III}

Distribution des types d'élevage camelins dans les différentes provinces du sud du Maroc

\begin{tabular}{|c|c|c|c|c|c|}
\hline Province & $\begin{array}{c}\text { Type } 1 \\
\text { Eleveurs de grands } \\
\text { troupeaux }\end{array}$ & $\begin{array}{c}\text { Type } 2 \\
\text { Eleveurs familiaux } \\
\text { citadins }\end{array}$ & $\begin{array}{c}\text { Type } 3 \\
\text { Eleveurs pluriactifs }\end{array}$ & $\begin{array}{c}\text { Type } 4 \\
\text { Eleveurs } \\
\text { sous-traitants }\end{array}$ & Total \\
\hline Boujdour & 65,0 & 5,0 & 15,0 & 15,0 & 100 \\
\hline Dakhla & 36,5 & 0,0 & 21,2 & 42,3 & 100 \\
\hline Essaouira & 0,0 & 100,0 & 0,0 & 0,0 & 100 \\
\hline Guelmim & 75,0 & 16,7 & 0,0 & 8,3 & 100 \\
\hline Laâyoune & 42,1 & 15,8 & 0,0 & 42,1 & 100 \\
\hline Ouarzazate & 7,1 & 92,9 & 0,0 & 0,0 & 100 \\
\hline Smara & 25,0 & 16,7 & 25,0 & 33,3 & 100 \\
\hline Tan-Tan & 73,7 & 0,0 & 10,5 & 15,8 & 100 \\
\hline Tata & 0,0 & 60,0 & 30,0 & 10,0 & 100 \\
\hline Total & 39,9 & 22,0 & 13,1 & 25,0 & 100 \\
\hline
\end{tabular}

Les chiffres en caractère gras sont significativement supérieurs et ceux en italiques significativement inférieurs $($ test du chi², $\mathrm{p}<0,05)$ 
(Faye et al., 2014 ; Bedda et al., 2015), pratique apparemment peu présente au Maroc.

\section{Origine tribale des éleveurs et stratégie de déplacement des dromadaires}

Cinquante-deux tribus ont été recensées au cours de cette étude ce qui représente un nombre important. Cela peut s'expliquer par le fait que, quelle que soit l'appartenance tribale des éleveurs, l'élevage des dromadaires au Maroc constitue une composante essentielle du mode de vie de la population sahraouie. Posséder un troupeau camelin est signe de notoriété dans la société et de fierté pour les éleveurs. Toutes les tribus sont plus ou moins impliquées dans cet élevage.

La mobilité des troupeaux de dromadaires est une donnée essentielle de la capacité des éleveurs à gérer les espaces pastoraux marqués par la rareté des ressources, comme cela a été décrit pour d'autres espèces (Butt et al., 2009). Dans notre échantillon, seuls $18 \%$ des troupeaux étaient sédentarisés. Ces animaux étaient utilisés en zones périurbaines, voire urbaines, soit pour assurer l'approvisionnement en lait des centres urbains comme cela a déjà été décrit au Maroc ou dans d'autres régions d'Afrique (Faye et al., 2003), soit uniquement à des fins de loisir dans les zones touristiques.

\section{Pratiques sanitaires}

Notre étude s'est limitée au relevé d'informations sur la vaccination antivariolique et contre l'ecthyma contagieux, ces maladies constituant une contrainte récurrente dans le sud du Maroc (El-Harrak et Loufti, 2000), ce qui expliquait le taux relativement élevé d'éleveurs pratiquant annuellement ou occasionnellement cette prévention $(67 \%$ au total). En revanche, si $85 \%$ des éleveurs ne faisaient pas appel à un médecin vétérinaire, c'est que l'usage de la pharmacopée traditionnelle reste prépondérant dans le milieu des chameliers (AntoineMoussiaux et al., 2007 ; Bornstein et Younan, 2013). Les conduites relatives aux bonnes pratiques prophylactiques telles que l'isolement des dromadaires malades ou la mise en quarantaine des animaux nouvellement acquis avant de les mettre en contact avec les dromadaires du troupeau sont quasi absentes chez les éleveurs chameliers au Maroc (Michel et al., 1997).

\section{Pratiques de supplémentation}

Les pratiques de supplémentation alimentaire s'appuient généralement sur diverses considérations que nous pouvons résumer en trois points : a) la supplémentation dépend de la disponibilité de sous-produits à des prix attractifs, b) elle s'impose en cas de contraintes fortes sur les ressources pastorales naturelles (déficit pluviométrique par exemple), et c) elle est encouragée en cas d'opportunité de vente du lait, donc pour augmenter la productivité des chamelles en lactation. Ces trois considérations induisent des stratégies diverses qui peuvent varier dans le temps.

En conséquence, des stratégies étaient différentes selon le type d'élevage. Les éleveurs sédentarisés autour des villes privilégient la supplémentation des laitières avec, si possible, les produits subventionnés (comme cela a été le cas de la pulpe de betterave dans le Sud marocain) alors que les éleveurs mobiles se focalisaient sur les animaux les plus vulnérables en saison sèche ou sur l'ensemble du troupeau, en cas de déficit pluviométrique, avec le disponible local (paille, écart de dattes) ou provenant des provinces du nord (céréales, maïs).

\section{Typologie des élevages}

Notre étude a permis de distinguer quatre types d'élevage en prenant en compte l'ensemble des critères descriptifs (taille du troupeau, statut et responsabilité du berger, supplémentation alimentaire, pratiques sanitaires, mobilité des troupeaux). Les types qui ressortaient de la classification automatique semblaient très liés à une variabilité interrégionale des systèmes d'élevage camelins comme l'indique le positionnement des provinces (variables supplémentaires) sur l'espace factoriel principal (figure 3) et le tableau de contingence croisant types d'élevage et province d'origine (tableau III).

Dans leur étude réalisée en Algérie, Harek et Bouhadad (2008) ont établi une «typologie structurelle » identifiant quatre modes d'élevage : les transhumants (20\%), les semi-transhumants (21\%), les semi-sédentaires $(18 \%)$ et les sédentaires $(21 \%)$. Le critère de mobilité des troupeaux camelins apparaît déterminant pour expliquer les types identifiés par classification automatique.

Cependant, une catégorisation des éleveurs sur ce seul critère apparaît de moins en moins pertinente. En effet, les éleveurs camelins s'adaptent à la fois aux nouveaux contextes climatiques et économiques par une tendance à la périurbanisation d'une partie du troupeau seulement comme évoqué plus haut, à des mobilités opportunistes, à une différenciation dans les lieux de vie et à une multiactivité afin de diversifier les sources de revenus (Correra et al., 2009).

Notre typologie indique en effet que la taille des troupeaux et leur mobilité n'étaient pas les seuls déterminants de la construction des types. L'organisation des hommes autour du troupeau est également un critère structurant (Michel et al., 1997). La multiactivité ou non du propriétaire, sa localisation (en ville ou non), la présence du ou des bergers, leur pouvoir plus ou moins autonome de décision, leur statut et leur proximité familiale avec le propriétaire, sont des critères qui peuvent permettre de comprendre le degré d'implication du propriétaire dans la conduite du troupeau et la délégation de sa gestion.

L'organisation des hommes autour du troupeau camelin fait apparaître une diversité d'objectifs des éleveurs et révèle une certaine mutation de l'élevage camelin (Breulmann et al., 2007). Les éleveurs ont en effet su s'adapter (contraintes climatiques, raréfaction des ressources naturelles, opportunités commerciales...) et profiter de nouvelles technologies telles que la téléphonie mobile. La sédentarisation partielle, transitoire ou définitive des éleveurs camelins (Faye, 2018) a fait évoluer leurs pratiques, leur organisation et leur rapport à l'espace en modulant leur mobilité, raccourcissant les distances à parcourir et les délais de réaction. Ainsi, grâce à la téléphonie mobile, les éleveurs peuvent rapidement connaître les événements qui ont lieu dans leurs troupeaux et réagir vite, mais ils peuvent aussi se permettre d'exercer d'autres activités en confiant la gestion journalière du troupeau à un berger, ou la responsabilité de la gestion de l'élevage à un contremaître (Michel et al., 1997).

Dans l'étude réalisée dans la zone périurbaine d'Agadez au Niger, Chaibou et Faye (2005) montrent que le critère le plus discriminant pour caractériser des systèmes camelins est la mobilité des troupeaux et les pratiques de complémentation. Ainsi, deux modes de conduite d'élevage existent sur la base du type de mobilité : transhumance en saison des pluies ou vrai nomadisme basé sur des mobilités incessantes ; cependant, un troisième mode semble émerger. Ce dernier concerne des éleveurs installés en zone périurbaine, pratiquant un système semi-intensif utilisant notamment des concentrés en complémentation et une mobilité réduite afin de répondre à l'approvisionnement de la ville d'Agadez en lait de chamelle (Faye et al., 2015). Le changement de régime de mobilité des troupeaux entraîne un changement des pratiques d'alimentation des animaux (Chaibou et Faye, 2005).

\section{CONCLUSION}

En dépit de la tradition nomade associée à l'élevage camelin, l'analyse des systèmes d'élevage camelins dans le sud du Maroc 
indique deux grandes tendances et un constat : a) une tendance à la sédentarisation, notamment vers les zones urbaines ou périurbaines pour répondre à la demande croissante en produits camelins (lait et viande) ; b) une tendance vers une gestion sous-traitante de la part des grands éleveurs par un transfert du pouvoir de décision vers les bergers et les contremaitres; et c) la faible implication des éleveurs dans les recommandations pratiques concernant les mesures prophylactiques, le recours à l'automédication témoignant du faible taux d'appel aux compétences vétérinaires dans tous les types d'élevage camelin.

Notre étude portant sur un échantillon géographique élargi à l'échelle du Maroc a permis d'appréhender la diversité spatiale des systèmes d'élevage camelin. Toutefois, des études complémentaires devraient être mises en œuvre sur la mobilité des troupeaux (ampleur, saisonnalité, localisation), notamment pour mieux aménager les parcours et instaurer les mesures prophylactiques nécessaires aux lieux de concentration des animaux. Il s'agirait aussi de mieux évaluer le potentiel de valorisation économique des produits camelins, l'une des principales contraintes au Maroc étant l'éloignement des lieux de production, concentrés au Sud, des centres de consommation, situés au Nord.

\section{Remerciements}

Cette étude a été réalisée et financée dans le cadre du projet PRAD n 16867 intitulé « Pathologies cutanées et métabolisme minéral chez le dromadaire ». Nos remerciements vont aux services vétérinaires marocains au niveau central, régional et local pour leur support technique au cours de la réalisation de cette étude ainsi qu'à $S$. Messad et X. Juanès du Cirad, Montpellier, France, pour leur soutien et assistance. A cela s'ajoutent nos remerciements aux relecteurs anonymes qui ont permis d'améliorer la présente publication

\section{Déclaration des contributions des auteurs}

AK a réalisé l'étude sur le terrain, les analyses statistiques et rédigé la première version de l'article ; $\mathrm{BF}$ a rédigé la seconde version de l'article, élaboré tous les graphiques et précisé les interprétations des analyses statistiques ; NST a contribué à la planification de l'étude ; MB a contribué à la révision critique du manuscrit ; tous les auteurs autorisent la soumission de la version finale en vue de sa publication

\section{Conflits d'intérêts}

L'étude a été réalisée sans aucun conflit d'intérêts.

\section{REFERENCES}

Abdallah H.R., Faye B., 2013. Typology of camel farming system in Saudi Arabia. Emir. J. Food Agric., 25 (4): 250-260, doi: 10.9755/ejfav25i4.15491

Adamou A., 2008. L'élevage camelin en Algérie : quel type pour quel avenir ? Sécheresse, 19 (4) : 253-260, doi : 10.1684/sec.2008.0149

Antoine-Moussiaux N., Faye B., Vias G.F., 2007. Tuareg ethnoveterinary treatments of camel diseases in Agadez area (Niger). Trop. Anim. Health Prod., 39: 83-89, doi: 10.1007/s11250-007-4404-1

Bedda H., Adamou A., Babelhadj B., 2015. Systèmes de production camelins au Sahara algérien : cas de la région de Ouargla. Algerian J. Arid Environ., 5 (1) : 115-127, doi : 10.12816/0045912

Bornstein S., Younan M., 2013. Significant veterinary research on the dromedary camels of Kenya: Past and Present. J. Camelid Sci., 6: 1-48

Breulmann M., Boer B., Wernery U., Wernery R., El-Shaer H., Alhadrami G., Gallacher D., et al., 2007. The camel, from tradition to modern times. Unesco, Doha, Qatar

Burger P., Ciani E., Faye B., 2019. Old world camels in a modern world A balancing act between conservation and genetic improvement. Anim. Genet. (Immunogenet., Mol. Genet. \& Function. Genom.), 1-15, doi: 10.1111/age. 12858

Butt B., Shortridge R., Winklerprins A., 2009. Pastoral herd management, drought coping strategies, and cattle mobility in Southern Kenya. Ann. Amer. Assoc. Geograph., 99 (2): 309-334, doi: 10.1080/00045600802685895

Chaibou M., Faye B., 2005. Herding strategies of camel husbandry in Agadez suburban area in Niger. Typological survey. Rev. Elev. Med. Vet. Pays Trop., 58 (4): 273-283, doi: 10.19182/remvt.9922

Correra A., Lefeuvre J.C., Faye B., 2009. Organisation spatiale et stratégie d'adaptation des nomades du parc national du Banc d'Arguin à la sécheresse. Sécheresse, 19 (4) : 245-251, doi: 10.1684/sec.2008.0150

El Abrak A., 2000. Encadrement sanitaire du cheptel camelin au Maroc. In : Maladies parasitaires et infectieuses du dromadaire (Ed. Dakkak A.). Actes Editions, Rabat, Maroc, 9 -14

El-Harrak M., Loufti C., 2000. Camel pox in the calf in Morocco. Identification of the isolated virus. Development and application to prophylaxis. Rev. Elev. Med. Vet. Pays Trop., 53 (2): 165-167, doi: 10.19182/remvt.9745

Everitt B., Landau S., Leese M., Stahl D. (Coord.), 2011. Cluster analysis, 5th Edn, Wiley, New York, USA, 330 p. (Ser. Probability and Statistics)

Faye B., 2016. Des dromadaires et des hommes au Moyen-Orient : Identité et modernité. Anthropol. Middle East, 11 (1) : 51-65, doi : 10.3167/ ame.2016.110106
Faye B., 2018. What future for camel pastoralism in the world? In: 5th Conf ISOCARD Recent advances in camelids biology, health and Production, Laayoune, Morocco, 12-15 Nov. 2018, 32-38

Faye B., Bengoumi M., Barkat A., 2003. Le développement des systèmes caméliens laitiers péri-urbains en Afrique. In : Atelier Int. Lait de chamelle en Afrique. FAO-Cirad-Karkara, Niamey, Niger, 5-8 nov. 2003, 115-125

Faye B., Chaibou M., Vias-Franck S.G., 2015. Adaptation of the pastoral farming systems: the example of the periurban dairy camel breeders in the area of Agadez (Niger). In: 4th Conf. ISOCARD Silk Road Camel: The Camelids, Main Stakes for Sustainable Development, Almaty, Kazakhstan, 8-12 June 2015, 315-317

Faye B., Jaouad M., Bhrawi K., Senoussi A., Bengoumi M, 2014. Camel farming in North Africa: Current state and prospects, Rev. Elev. Med. Vet. Pays Trop. 67 (4): 213-221, doi :10.19182/remvt.20563

Faye B., Lefèvre P.C., Lancelot R., Quirin R., 1994. Ecopathologie animale: méthodologie; applications en milieu tropical. INRA CIRAD, Versailles, France, 119 p.

Faye B., Senoussi H., Jaouad M., 2017. Le dromadaire et l'oasis : du caravansérail à I'élevage périurbain. Cah. Agric., 26 (1), doi: 10.1051/ cagri/2017005

Harek D., Bouhadad R., 2008. La diversité des élevages camelins dans la région du Hoggar (Wilaya de Tamanrasset), In : Colloq. Int. Développement durable des productions animales : enjeux, évaluation et perspectives, Alger, Algérie, 30 nov. 2013, 20-21

Michel J.F., Bengoumi M., Bonnet P., Hidane K., Zro K., Faye B., 1997. Typology of dromedary production systems in Laâyoune province, Morocco. Rev. Elev. Med. Vet. Pays Trop., 50 (4): 313-32, doi : 10.19182/remvt.9563

Ouassat M., Achaabane R., 1991. Identification des principales races camelines. In : «Etudes et recherches appliquées sur l'élevage camelin. Rapport de synthèse. Gerdec, Rabat, Maroc, 1-12

Oulad Belkhir A, Chehma A., Faye B., 2013. Phenotypic variability of two principal Algerian camel's populations (Targui and Sahraoui). Emir. J. Food Agric., 25 (3): 231-237, doi: 10.9755/ejfa.v25i3.15457

Salmi Ch., Jaouad M., Faye B., Haouat F., 2016. Typologie des éleveurs camelin au sud-est tunisien en vue de leurs performances économiques. In Actes Colloq. Développement socio-économique et dynamique des sociétés rurales : Pluralité d'acteurs, gestion des ressources et développement Territorial, Zarzis, Tunisie, 3-5 mai 2016. Rev. Régions Arides 44 (1) : 209214

Titaouine M., Mohamdi H., Deghnouche K., 2015. Diagnostic de la situation des éleveurs de dromadaire dans le Sud-Est algérien (la Willaya d'El-Oued, Algérie). Rencontres Rech. Rumin., 22-364 


\section{Summary}

Kamili A., Faye B., Tligui N.S., Bengoumi M. Typology of camel farming systems in the south of Morocco

Although the national authorities under the Green Morocco Plan support camel farming, it is still relatively poorly known in Morocco. The objective of this study was to provide a description of the types of camel farms in a large portion of the southern part of the country, characterized by its high concentration of camel farms. To do this, as part of a sanitary survey, 168 camel farmers from nine provinces of the south of Morocco were interviewed on their production systems. The 168 camel herds represented 11,426 camels, i.e. about $10 \%$ of the camel population in the area. Finally, a data table including 168 camel farms described by twenty active variables (size and structure of the herd, management organization and decision-making power, importance of mobility, marketing, feed supplementation practices and health prevention) and two illustrative variables (status and region of origin of the respondent) were subjected to a multivariate analysis (multiple correspondence analysis and automatic classification). The analysis identified four breeder types: i) breeders with large traditional dairy herds, ii) urban farmers with small herds, iii) opportunistic multiactive breeders, and iv) breeders with occasional multiactivity, low market integration, and subcontracting their camel farming activity. This typology appears to be very closely related to the breeders' region of origin, showing some regional specificity.

Keywords: Camelus dromedarius, typology, animal husbandry, livestock management, Morocco

\section{Resumen}

Kamili A., Faye B., Tligui N.S., Bengoumi M. Tipología de los sistemas de cría de camellos en el sur de Marruecos

A pesar de contar con el apoyo de las autoridades nacionales en el marco del Plan Marruecos Verde, la cría de cameIlos en Marruecos es aún relativamente desconocida. El presente estudio tuvo por objetivo obtener una descripción de los tipos de cría de camellos en una vasta porción del sur del país, caracterizada por su gran concentración de fincas de camellos. Para esto, en el marco de una encuesta sanitaria, se entrevistaron 168 criadores de camellos pertenecientes a nueve provincias del sur de Marruecos, sobre sus sistemas de producción. Los 168 hatos de camellos representaron 11426 dromedarios, o sea aproximadamente $10 \%$ de la población de camellos de esta zona. Al finalizar, un cuadro incluyendo 168 criaderos de camellos descritos por 20 variables activas (tamaño y estructura del hato, organización de la gestión y del poder de decisión, amplitud de la movilidad, comercialización, practicas de complementación alimenticia y de prevención sanitaria) y dos variables ilustrativas (situación del encuestado y región de origen) se sometió a un análisis multivariado (análisis de correspondencias múltiples y clasificación automática). El análisis permitió identificar cuatro tipos de criadores: a) aquellos con grandes hatos lecheros tradicionales, b) citadinos con pequeños hatos, c) criadores oportunistas con actividad múltiple, y d) criadores con actividad múltiple ocasional, pobremente integrados al mercado y subcontratando la actividad de cría. Esta tipología parece estar relacionada con la región de origen de los criadores, indicando una cierta especificidad regional.

Palabras clave: Camelus dromedarius, tipología, ganadería, manejo del ganado, Marruecos 